\title{
Landscape Plants of Recep Tayyip Erdoğan University, Zihni Derin Campus \\ (Rize-Turkey) $^{[*]}$
}

\author{
Ahmed Nazmi BAL ${ }^{1}$ Gökhan ABAY ${ }^{2}$ \\ ${ }^{1}$ Landscape Architecture, Trabzon, Turkey. (D): https://orcid.org/0000-0001-5565-0535 \\ ${ }^{2}$ Recep Tayyip Erdoğan Üniversitesi, Peyzaj Mimarlığı Bölümü, Fener Yerleşkesi, 53100/ Rize, Türkiye. D: https://orcid.org/0000-0002-5512-337X
}

Received date: 25.01 .2019

Accepted date: 11.03 .2019

How to cite: Bal, A.N. \& Abay, G. (2019). Landscape plants of Recep Tayyip Erdoğan University, Zihni Derin campus (Rize-Turkey). J. Anatolian Env.
and Anim. Sciences, 4(1), 11-15. Doi: https://doi.org/10.35229/jaes.517526
Attf yapmak için: Bal, A.N. \& Abay, G. (2019). Recep Tayyip Erdoğan Üniversitesi, Zihni Derin yerleşkesi peyzaj bitkileri (Rize-Türkiye).
Anadolu Çev. ve Hay. Dergisi, 4(1), 11-15. Doi: https://doi.org/10.35229/jaes.517526

Abstract: A survey of gymnosperms and angiosperms used in landscape design in Zihni Derin Campus of Recep Tayyip Erdoğan University was carried out between the years 2016-2017. The plants in the campus comprise 155 taxa belonging to 98 genera and 50 families. Within the angiosperms; Rosaceae has the highest number of taxa (13.9\%). Primulaceae with $5.7 \%$ and Asparagaceae with $4.9 \%$ followed it, respectively. The others have a percentage of $75.5 \%$ of the landscape plant species belonging to angiosperms. If we look at the gymnosperms; Cupressaceae has the highest number of taxa (69.7\%). Pinaceae with $27.3 \%$ followed it. The other and last family Cycadaceae has a percentage of $3 \%$ within the needle leaves. From the plants which consist of 155 taxa, 125 are in tree and shrub form, and the rest of them (30 taxa) are herbaceous.

Keywords: Angiospermae, gymnospermae, landscape plants, Rize, Turkey, Zihni Derin Campus.

\section{Recep Tayyip Erdoğan Üniversitesi, Zihni Derin Yerleşkesi Peyzaj Bitkileri (Rize-Türkiye)}

Öz: 2016-2017 yılları arasında Recep Tayyip Erdoğan Üniversitesi Zihni Derin Yerleşkesinde peyzaj tasarımında kullanılan tohumlu bitkiler üzerine bir araştırma gerçekleştirilmiştir. Kampüsteki bitkiler, 98 cins ve 50 familyaya ait 155 taksonu kapsamaktadır. Kapalı tohumlu bitkiler içinde Rosaceae en fazla taksona sahiptir (\% 13.9). Primulaceae \% 5.7 ve Asparagaceae \% 4.9 ile sırasıly izlemiştir. Diğerlerinin kapalı tohumlulara ait peyzaj bitki türleri içerisindeki oranı \% 75.5 tur. Açık tohumlulardan; Cupressaceae en fazla sayıda taksona (\% 69.7) sahiptir. Pinaceae \% 27.3 ile onu izlemiştir. Cycadaceae'nin açık tohumlular oranı \% 3 tür. 155 taksondan 125'i ağaç ve çalı formunda, geri kalan 30 takson ise otsudur.

Anahtar sözcükler: Kapalı tohumlular, açık tohumlular, peyzaj bitkileri, Rize, Türkiye, Zihni Derin Yerleşkesi. 


\section{INTRODUCTION}

Plants that have different appearance according to different seasons and years constitute the main material of the plant design. It is important to know some basic features such as aesthetic and functional principals of the plants used apart from their botanical and ecological desires while making landscape architecture design studies. Revealing these characteristics of the plants by taking into consideration the harmonization with the environment, environmental regulations and sustainable studies (Zencirkıran, 2013).

In university campuses, an important part of urban habitats, the increase of physical spaces with the increase of students and staff over time requires the open and green areas to be designed better. Having a well-designed and planted campus area is important for the life quality of both studies and the staff (Çorbac1 et al., 2005).

Up to now, there are many floristic studies made within the boundaries of university campuses in our country. These can be summarized as; Akdeniz University Campus Flora (Ünal \& Gökçeoğlu, 2003), Kırıkkale University Campus Flora (Nugay et al., 2007), Beytepe Campus (Ankara) Flora (Mutlu et al., 2008), Gaziantep University Campus Flora (Uma et al., 2008), Natural Plant Species of Süleyman Demirel University Campus (Isparta-Türkiye) (Fakir et al., 2009), Niğde University Campus Flora (Başköşe et al., 2012), İnönü University Campus Flora (Malatya) (Mutlu \& Karakuş, 2012), Ordu University Campus Flora (Deveci et al., 2012), Vascular Plants of Buca Education Faculty Campus (İzmir) (Ugulu et al., 2012), Boğaziçi University Flora Project (BÜF) (Özhatay et al., 2015) and Dumlupınar University Central Campus (Kütahya) Flora (Tatli et al., 2002). On the other hand, studies on landscaping and ornamental plants in various universities campuses are less than expected. Some examples are; Samsun Ondokuz Mayıs University Kurupelit Ornamental Plants (Özen et al., 1998) and Mustafa Kemal University Tayfur Ata Sökmen Campus (Hatay) Ornamental Plants (Altay, 2012).

The floristic structure of the largest campus "Zihni Derin", belonging to the Recep Tayyip Erdoğan University founded in 2006 and one of the fastest developing universities having many settlements around the main campus and the provinces, is under constant change with the settlement and landscaping studies, with the establishment of the central campus many local and foreign landscaping plants were added to pre-existing taxa in the area.

There are no studies published on important plant species in terms of floristic and landscape in the campus area. In this study, the landscape plants of the area were identified and listed according to tree, shrub and herbaceous forms. By the determination of plant taxa having the value of the landscape, we will take a step in the extraction of the plant composition of the mentioned area leading to illuminate the natural studies that will be done in the campus area.

\section{MATERIAL and METHODS}

The material of this work, carried out between the years of 2016-2017, is the landscape plants in the woody and herbaceous forms of Recep Tayyip Erdoğan University Zihni Derin Campus. The plants used in landscape design within the boundaries of the campus were photographed by making field trips during the leafy and flowering periods and tried to be identified in the field. The plants which couldn't be identified in the field were diagnosed with the help of related flora works such as (Pamay, 1992-1993; Taverne, 1995; Bettini, 2000; Ceylan, 2004; Foulis et al., 2004; Güngör et al., 2007; Ebcioğlu, 2008; Mamıkoğlu, 2008; Şengönül \& Y1lmaz, 2008; Tuzlac1, 2007, 2010; Yücel, 2012a, 2012b; Efe \& Yılmaz, 2013; Zencirkıran, 2013; Akkemik, 2014a, 2014b; Ekren, 2014). Specimens that couldn't be determined were given as "sp." in the text.

Plant taxa are classified according to their forms such as woody (tree and shrub) or herbaceous and given in alphabetical order. http://www.theplantlist.org/ and https://scholar.google.com.tr/ internet sites were used to find the Latin names and authors of the taxa. Following this, families belonging to taxa are given.

\section{RESULTS AND DISCUSSION}

\section{Trees and Shrubs \\ Gymnosperms}

Cedrus atlantica (Endl.) Manetti ex Carrière cv. 'Glauca' (Pinaceae)

Cedrus deodora (Roxb. ex D. Don) G. Don (Pinaceae)

Chamaecyparis lawsoniana (A.Murray bis) Parl.

(Cupressaceae)

Chamaecyparis lawsoniana cv. 'Ellwoodii' (Cupressaceae)

Chamaecyparis pisifera (Siebold \& Zucc.) Endl. cv. 'Boulevard' (Cupressaceae)

Cryptomeria japonica (Thunb. ex L. f.) D. Don (Cupressaceae)

Cupressus arizonica Greene (Cupressaceae)

Cupressus arizonica Greene cv. 'Gold' (Cupressaceae)

Cupressus macrocarpa Hartw. cv. 'Goldcrest' (Cupressaceae)

Cycas revoluta Thunb. (Cycadaceae)

Juniperus chinensis L. cv. 'Pfitzeriana Glauca' (Cupressaceae)

Juniperus communis L. cv. 'Depressa Aurea' (Cupressaceae)

Juniperus communis L. cv. 'Hibernica' (Cupressaceae)

Juniperus horizontalis Moench cv. 'Aurea' (Cupressaceae)

Juniperus squamata Buch.-Ham. ex D. Don cv. 'Blue Carpet' (Cupressaceae) 
Juniperus virginiana L. (Cupressaceae)

Juniperus virginiana L. cv. 'Skyrocket' (Cupressaceae)

Picea abies (L.) H.Karst. (Pinaceae)

Picea glauca (Moench) Voss (Pinaceae)

Picea orientalis (L.) Peterm. (Pinaceae)

Pinus griffithii (Hook.f.) Parl. cv. 'Glauca' (Pinaceae)

Pinus pinaster Aiton (Pinaceae)

Pinus pinea L. (Pinaceae)

Pinus radiata D.Don (Pinaceae)

Thuja occidentalis L. (Cupressaceae)

Thuja occidentalis L. cv. 'Golden Smaragd' (Cupressaceae)

Thuja occidentalis L. cv. 'Rheingold' (Cupressaceae)

Thuja occidentalis L. cv. 'Smaragd Aurea' (Cupressaceae)

Thuja occidentalis L. cv. 'Smaragd' (Cupressaceae)

Thuja occidentalis L. cv. 'Tiny Tim' (Cupressaceae)

Platycladus orientalis (L.) Franco (Cupressaceae)

Thuja plicata Donn ex D.Don cv. 'Gold' (Cupressaceae)

Thujopsis dolobrata (L.f.) Siebold \& Zucc. (Cupressaceae)

\section{Angiosperms}

\section{Trees and Shrubs}

Abelia grandifolia Villarreal (Caprifoliaceae)

Acer negundo f. flamingo Geerinck (Sapindaceae)

Acer palmatum f. atropurpureum (Van Houtte) G.Nicholson (Sapindaceae)

Acer platanoides f. globosum (G.Nicholson) Schwer. (Sapindaceae)

Acer platanoides L. cv. 'Crimson King' (Sapindaceae)

Ailanthus altissima (Mill.) Swingle (Simaroubaceae)

Albizia julibrissin Durazz. (Fabaceae)

Aucuba japonica Thunb. 'Variegata' (Garryaceae)

Azalea japonica 'Amoena' (Ericaceae)

Azalea japonica 'Homebush' (Ericaceae)

Azalea japonica 'Pink King' (Ericaceae)

Berberis thunbergii DC. var. atropurpurea Chenault (Berberidaceae)

Betula pubescens Ehrh. (Betulaceae)

Buxus sempervirens 1. var. rotundifolia Baill. (Buxaceae)

Callistemon citrinus (Curtis) Skeels (Myrtaceae)

Camellia japonica L. (Theaceae)

Camellia sinensis (L.) Kuntze (Theaceae)

Campsis radicans (L.) Seem. (Bignoniaceae)

Cercis siliquastrum L. (Fabaceae)

Citrus limon (L.) Osbeck (Rutaceae)

Citrus reticulata Blanco (Rutaceae)

Cordyline australis (G.Forst.) Endl. (Asparagaceae)

Cordyline indivisa (G.Forst.) Endl. (Asparagaceae)

Cordyline indivisa (G.Forst.) Endl. cv. 'Aureavariegata' (Asparagaceae)

Cordyline indivisa (G.Forst.) Endl. cv. 'Red Star'

(Asparagaceae)

Corylus avellana L. (Betulaceae)

Cotinus coggygria Scop. (Anacardiaceae)

Cotoneaster lacteus W.W.Sm. (Rosaceae)
Cydonia oblonga Mill. (Rosaceae)

Deutzia gracilis Siebold \& Zucc. (Hydrangeaceae)

Eriobotrya japonica (Thunb.) Lindl. (Rosaceae)

Euonymus japonicus Thunb. cv. 'Microphyllus' H.Jaeger (Celastraceae)

Euonymus japonicus f. aureovariegatus (Regel) Rehder (Celastraceae)

Euonymus japonicus Thunb. var. aureovariegatus Regel (Celastraceae)

Euonymus japonicus Thunb. cv. 'Luna' (Celastraceae)

Fatsia japonica (Thunb.) Decne. \& Planch. (Araliaceae)

Ficus carica L. (Moraceae)

Forsythia $\times$ intermedia Zabel (Oleaceae)

Hedera helix L. (Araliaceae)

Hedera helix f. aureovariegata (Weston) P.D.Sell (Araliaceae)

Hydrangea macrophylla (Thunb.) Ser. (Hydrangeaceae)

Ilex aquifolium L. cv. 'Aurea Variegata' (Aquifoliaceae)

Jasminum fruticans L. (Oleaceae)

Jasminum officinale L. (Oleaceae)

Juglans regia L. (Juglandaceae)

Laurus nobilis L. (Lauraceae)

Ligustrum japonicum Thunb. (Oleaceae)

Ligustrum vulgare cv. 'Aureum' (Oleaceae)

Liquidambar styraciflua L. (Altingiaceae)

Lonicera caprifolium L. (Caprifoliaceae)

Loropetalum chinense (R. Br.) Oliv. (Hamamelidaceae)

Loropetalum chinense cv. 'Rubrum' (Hamamelidaceae)

Magnolia $\times$ soulangeana Soul.-Bod. (Magnoliaceae)

Magnolia grandiflora L. (Magnoliaceae)

Mespilus germanica L. (Rosaceae)

Morus nigra L. cv. 'Pendula' (Moraceae)

Nandina domestica Thunb. (Berberidaceae)

Nandina domestica Thunb. cv. 'Firepower' (Berberidaceae)

Nerium oleander L. (Apocynaceae)

Nerium oleander L. cv. 'Aurea Variegata' (Apocynaceae)

Parthenocissus quinquefolia (L.) Planch. (Vitaceae)

Philadelphus coronarius L. (Hydrangeaceae)

Phoenix canariensis Chabaud (Arecaceae)

Phormium tenax J.R.Forst. \& G.Forst. cv. 'Maori Maiden' (Asphodelaceae)

Phormium tenax J.R.Forst. \& G.Forst. cv. 'Variegatum' (Asphodelaceae)

Photinia $\times$ fraseri Dress cv. 'Red Robin' (Rosaceae)

Pittosporum tobira (Thunb.) W.T.Aiton cv. 'Nana'

(Pittosporaceae)

Pleioblastus simonii (Carrière) Nakai (Poaceae)

Prunus laurocerasus L. (Rosaceae)

Prunus domestica L. (Rosaceae)

Prunus serrulata Lindl. cv. 'Kanzan' (Rosaceae)

Pyracantha coccinea M.Roem. cv. 'Nana' (Rosaceae)

Pyracantha coccinea M.Roem. cv. 'Orange Glow'

(Rosaceae)

Pyrus communis L. (Rosaceae) 
Pyrus sp. (Rosaceae)

Rhododendron molle subsp. japonicum (A.Gray) Kron (Ericaceae)

Robinia pseudoacacia L. (Fabaceae)

Rosa sp. (Rosaceae)

Rosmarinus officinalis L. (Lamiaceae)

Rubus sp. (Rosaceae)

Salix alba L. (Salicaceae)

Salix babylonica L. (Salicaceae)

Salix caprea L. (Salicaceae)

Spiraea $\times$ bumalda Burv. (Rosaceae)

Spiraea $\times$ vanhouttei (Briot) Zabel (Rosaceae)

Tecoma capensis (Thunb.) Lindl. (Bignoniaceae)

Trachycarpus fortunei (Hook.) H.Wendl. (Arecaceae)

Viburnum tinus L. (Adoxaceae)

Vitis vinifera L. (Vitaceae)

Washingtonia filifera (Linden ex André) H.Wendl. ex de Bary (Arecaceae)

Weigela floribunda C.A.Mey. (Caprifoliaceae)

Wisteria sinensis (Sims) Sweet (Fabaceae)

\section{Herbaceous Plants}

Agave americana L. (Asparagaceae)

Aloe vera (L.) Burm.f. (Asphodelaceae)

Begonia cucullata Willd. (Begoniaceae)

Canna $\times$ generalis L.H. Bailey \& E.Z. Bailey (Cannaceae)

Carex sp. (Cyperaceae)

Chrysanthemum sp. (Asteraceae)

Coreopsis lanceolata L. (Asteraceae)

Cortaderia selloana (Schult. \& Schult.f.) Asch. \& Graebn. (Poaceae)

Cortaderia selloana (Schult. \& Schult.f.) Asch. \& Graebn. cv. 'Gold Band' (Poaceae)

Cyclamen sp. (Primulaceae)

Euryops pectinatus (L.) Cass. (Asteraceae)

Hemerocallis fulva (L.) L. (Asphodelaceae)

Iris laevigata Fisch. (Iridaceae)

Jacobaea maritima (L.) Pelser \& Meijden (Asteraceae)

Lampranthus multiradiatus (Jacq.) N.E.Br. (Aizoaceae)

Lavandula angustifolia Mill. (Lamiaceae)

Ophiopogon japonicus (Thunb.) Ker Gawl. (Asparagaceae)

Oxalis floribunda Lehm. (Oxalidaceae)

Primula vulgaris Huds. cv. 'Dark Red' (Primulaceae)

Primula vulgaris Huds. cv. 'Orange' (Primulaceae)

Primula vulgaris Huds. cv. 'Purple' (Primulaceae)

Primula vulgaris Huds. cv. 'Red' (Primulaceae)

Primula vulgaris Huds. cv. 'White' (Primulaceae)

Primula vulgaris Huds. cv. 'Yellow' (Primulaceae)

Santolina chamaecyparissus L. (Asteraceae)

Tulipa sp. (Liliaceae)

Verbena hybrida Groenl. \& Rumpler (Verbenaceae)

Vinca major L. (Apocynaceae)

Vinca major L. cv. 'Aureo-Variegata' (Apocynaceae)

Zantedeschia aethiopica (L.) Spreng. (Araceae)
There are 155 landscape plants including 50 families and 98 species within the university campus. These are woody (tree and shrub) and herbaceous forms. It was observed that some of the plants were naturally present in the country, while the rest were used exotically, in plant design studies.

The vascular plant taxa found in the campus belong to Spermatophyta division. Thirty-three of them belong to Gymnospermae, 122 belong to Angiospermae subdivision. According to the results, the first three families with the highest numbers of taxa are Rosaceae (13.9\%), Primulaceae (5.7\%) and Asparagaceae (4.9\%). Within the Gymnosperms, Cupressaceae is the family with the highest number of taxa and $69.7 \%$. Pinaceae is the second with $27.3 \%$, followed by Cycadaceae with a percentage of $3 \%$ in coniferous.

\section{CONCLUSIONS}

Since the newly introduced plant species and the floristic structure are under constant change in terms of settlement and environmental regulation in the campus area, it is important to determine the natural flora elements before the construction of the campus area is fully completed.

\section{ACKNOWLEDGEMENTS}

We are very grateful to the Scientific and Technological Research Council of Turkey TÜBİTAK (Project Number: 1919B011602921) for financial support.

\section{REFERENCES}

Akkemik, Ü. (Editör). (2014a). Türkiye'nin doğal-egzotik ağaç ve çalıları I, Gymnospermler-Angiospermler $(A-G)$. Orman Genel Müdürlüğü Yayınları, Ankara, $736 \mathrm{~s}$.

Akkemik, Ü. (Editör). (2014b). Türkiye'nin doğal-egzotik ăgaç ve çalıları II, Angiospermler (H-Z). Orman Genel Müdürlüğü Yayınları, Ankara, 680s.

Altay, V. (2012). Mustafa Kemal Üniversitesi Tayfur Ata Sökmen Kampüsü (Hatay)'nün Süs Bitkileri. Karadeniz Fen Bilimleri Dergisi, 2(6), 11-26.

Anonymous. (January 2018). Google Akademik. [Online]. Available: https://scholar.google.com.tr/

Anonymous. (January 2018). The plant list. A working list of a plant species. [Online]. Available: http://www.theplantlist.org/

Başköşe, İ., Paksoy, M.Y. \& Savran, A. (2012). The flora of Niğde University campus area and Akkaya dam lake environments (Niğde/Turkey). Biological Diversity and Conservation, 5(3), 82-97. 
Bettini, A. (2000). II Millepiante, Italyan fidanlık bitkileri el kitabı. G.T. Piante srl ve Euro Plant Tarım san. Tic. Ltd. Şti., İtalya, 205s.

Ceylan, G. (2004). Dış mekân süs bitkileri ve peyzajda kullanımları. Flora Yayınları, İstanbul, 216s.

Çorbacı, Ö.L., Gülez, S. \& Topay, M. (2005). ZKÜ Merkez kampüsü 1sı merkezi yolu ve çevresi peyzaj ve rekreasyon projesi. ZKU Bartın Orman Fakültesi Dergisi, 7(7), 24-34.

Deveci, M., Bayrak Özbucak, T. \& Demirkol, G. (2012). Ordu Üniversitesi kampüs alanı florasının tespiti. Akademik Ziraat Dergisi, 1(2), 107-116.

Ebcioğlu, N. (2008). Su bitkileri. İnkılap Kitabevi Baskı Tesisleri, İstanbul, 136s.

Efe, A. \& Yılmaz, H. (2013). İstanbul ormanlarının doğal ă̆aç ve çalıları. Türkiye Ormancılar Derneği, Dönmez Ofset, Ankara, 285s.

Ekren, E. (2014). Peyzaj bitkileri ve özellikleri. Kitap Matbaacılık Sanayi ve Ticaret Ltd. Şti., İstanbul, $160 \mathrm{~s}$.

Fakir, H., Babalık, A.A. \& Karatepe, Y. (2009). Süleyman Demirel Üniversitesi kampüsünün doğal bitki türleri (Isparta-Türkiye). Süleyman Demirel Üniversitesi Fen Bilimleri Enstitüsü Dergisi, 13(1), 33-39.

Foulis, L., \& 20 co-authors (Editors). (2004). Botanica, The illustrated $A-Z$ of over 10,000 garden plants and how to cultivate them. h.f. Ullmann publishing GmbH, Germany, 1020p.

Güngör, İ., Atatoprak, A., Özer, F., Akdağ, N. \& Kandemir, N.í. (2007). Bitkilerin dünyası, bitki tanitım detayları ile fidan yetiştirme esaslart. Lazer Ofset Matbaa Tesisleri Sanayi ve Ticaret Ltd. Şti., Ankara, 382s.

Mamıkoğlu, N.G. (2008). Türkiye'nin ăgaçları ve çalıları. NTV Yayınları, Doğuş Grubu İletişim Yayıncılık ve Ticaret A.Ş., İstanbul, 727s.

Mutlu, B., Erik, S. \& Tarikahya, B. (2008). New contributions to the flora of Beytepe campus (Ankara) and floristic comparison with neighboring floras and other campus floras. Hacettepe Journal of Biology and Chemistry, 36(3), 181-195.

Mutlu, B. \& Karakuş, Ş. (2012). İnönü Üniversitesi kampüs floras1 (Malatya). 21. Ulusal Biyoloji Kongresi, Bildiriler Kitabl, 03-07 Eylül 2012, İzmir, s, 656.

Nugay, Ö.Z., Duran, A. \& Doğan, B. (2007). Kırıkkale Üniversitesi kampüs florası. SÜ Fen Ed Fak Derg, 30, 79-92.

Özen, F., Kılınç, M. \& Uz, S. (1998). Samsun Ondokuz Mayıs Üniversitesi Kurupelit kampüs alanındaki süs bitkileri. Ekoloji Çevre Dergisi, 7(27), 26-30.

Özhatay, N., Akalın, E., Koçyiğit, M. \& Çokoy, A. (2015). Boğaziçi Üniversitesi florası Projesi (BÜF), 1. Ara rapor.
Pamay, B. (1992). Bitki materyali I Ăgaç ve ăgaçcıklar. Uycan Matbaas1. İstanbul, 80s.

Pamay, B. (1993). Bitki materyali II Çiçekli çalılar, sarmaşıklar, kaktüsler, saz ve kamışlar. Orhan Ofset, İstanbul, 244s.

Şengönül, S. \& Yılmaz, H. (2008). Atatürk arboretumu ă̆aç ve çalıları. Atatürk Arboretumu Yayını, İstanbul, 486s.

Tatlı, A., Tel, A. Z. \& Emre, S. (2002). Dumlupınar Üniversitesi merkez kampüsü (Kütahya) florası, Dumlupınar Üniversitesi Fen Bilimleri Dergisi, (10. Y1l Özel Sayısı), 3, 1-16.

Taverne, Y. (Editör). (1995). Garden shrubs, flowers \& plants. Magna Books, England, 75p.

Tuzlacı, E. (2007). Dekoratif Türkiye bitkileri. Melisa Matbaac1l1k, İstanbul, 560s.

Tuzlacı, E. (2010). Türkiye'nin bahçe bitkileri ve kent çiçekleri. Golden Medya Matbaacılık ve Ticaret A.Ş., İstanbul, 885s.

Ugulu, I., Dogan, Y. \& Kesercioğlu, T. (2012). The vascular plants of Buca faculty of education campus (İzmir): Contribution to educational practices. EurAsian Journal of BioSciences, 6, 11-23.

Uma, M.M., Yayla, F. \& Zeynalov, Y. (2008). Gaziantep Üniversitesi kampüs floras1. 19. Ulusal Biyoloji Kongresi, Bildiriler Kitabl, 23-27 Haziran 2008, Trabzon, s, 472.

Ünal, O. \& Gökçeoğlu, M. (2003). Akdeniz Üniversitesi kampüs florası (Antalya-Türkiye). Akdeniz Üniversitesi Ziraat Fakültesi Dergisi, 16(2), 143154.

Yücel, E. (2012a). Çiçekler ve yerörtücüler. Türmatsan Organize Matbaacılık San. Ltd. Şti. Eskişehir, 352s.

Yücel, E. (2012b). A ̈̆açlar ve çalılar I. Türmatsan Organize Matbaacılık San. Ltd. Şti. Eskişehir, 277s.

Zencirkıran, M. (2013). Peyzaj bitkileri I (Açık tohumlu bitkiler-Gymnospermae). Nobel Akademik Yayıncılık Eğitim Danışmanlık Tic. Ltd. Şti., Ankara, 475s.

\section{*Corresponding author's:}

Prof. Dr. Gökhan ABAY

Recep Tayyip Erdoğan Üniversitesi, Peyzaj Mimarlığ Bölümü, Fener Yerleşkesi, 53100/ Rize, Türkiye.

E-mail: gokhanabay@gmail.com

ORCID: https://orcid.org/0000-0002-5512-337X 